\title{
Fermions Tunneling from Charged anti-de Sitter Black Holes
}

\author{
M. Sharif *and Wajiha Javed ${ }^{\dagger}$ \\ Department of Mathematics, University of the Punjab, \\ Quaid-e-Azam Campus, Lahore-54590, Pakistan.
}

\begin{abstract}
We study Hawking radiation as a phenomenon of tunneling through event horizons of charged torus-like as well as dilaton black holes involving cosmological constant based on Kerner and Mann's formulation. We obtain tunneling probabilities as well as Hawking's emission temperature of outgoing charged particles by applying the semiclassical WKB approximation to the general covariant Dirac equation. The graphical behavior of Hawking temperature and horizon radius is investigated. We find results consistent with those already given in literature.
\end{abstract}

Keywords: Quantum tunneling; Torus-like and dilaton black holes. PACS numbers: 04.70.Dy; 04.70.Bw; 11.25.-w

\section{Introduction}

Hawking [1] demonstrated that the surface area of event horizon of a black hole $(\mathrm{BH})$ can never decrease with time. This led to an interesting new era of research in $\mathrm{BH}$ physics. Bekenstein 22 proved that the $\mathrm{BH}$ entropy is proportional to its horizon area. This made a foundation of the correspondence between the classical as well as BH laws of thermodynamics. Thus,

*msharif.math@pu.edu.pk

†wajihajaved84@yahoo.com 
$\mathrm{BH}$ refers to as a thermodynamic substance having entropy corresponding to its horizon area, a temperature related to its surface gravity $\kappa$ and an internal energy comparable to its mass. According to Hawking [3], a BH is not sufficiently black but actually discharges radiation named as Hawking radiation.

Semiclassically, quantum field distribution in the environment of a $\mathrm{BH}$ manifests that it emits black body radiation with thermal spectrum at Hawking temperature $T_{H}=\frac{\kappa}{2 \pi}$ and entropy $S=A / 4$. Quantum gravity encourages the phenomenon of information loss from BHs, according to which the Hawking temperature increases as the mass of the $\mathrm{BH}$ diminishes. Thus, a $\mathrm{BH}$ would radiate repeatedly some of its mass through Hawking radiation, leading to increase in Hawking temperature. Since BH radiation is a continuous process, so with the passage of time, finally it evaporates completely. The final stage of the evaporated $\mathrm{BH}$ has considerably small size of the order of Planck length. Consequently, the ultimate stage of evaporation can only be illustrated by a quantum gravity theory.

The radiation spectrum of BHs has been discussed by using various techniques. Damour and Ruffini [4] provided unification of quantum mechanics and general relativity to analyze Hawking's radiation progression. Kraus and Wilczek [5] extended the tunneling procedure to investigate the Hawking radiation by discussing correlations between incoming particles and the radiation. They considered self-gravitational effects of radiation and found that particles no longer move along geodesics and corresponding radiation spectrum is not precisely thermal. Keski-Vakkuri and Kraus [6] developed a tunneling algorithm to study the radiation spectrum based on the WKB approximation together with complex time path techniques. This algorithm is used to calculate the tunneling probabilities for systems with time dependent background. Later, this method was used by Parikh and Wilczek [7]-[9] in order to formulate another tunneling approach to investigate the tunneling radiation features of the Schwarzschild and Reissner-Nordström (RN) BHs.

Following Parikh and Wilczek semiclassical tunneling method, there has been a debate for tunneling of particles across the horizon either massive or massless as well as charged or uncharged. Hemming and Keski-Vakkuri [10] explored the radiation spectrum from anti-de Sitter BH, while Medved [11] studied from a de Sitter BH. Zhang and Zhao [12, 13] investigated radiation phenomenon from an axisymmetric BH. These results hold the Parikh and Wilczek conclusion, i.e., the true Hawking radiation spectrum is not purely thermal due to the significance of self-gravitational effects. Kerner and Mann 
[14] proposed a method to examine fermion's tunneling by extending the idea that the Hawking radiation may emit fermions. For this purpose, they used the general Dirac equation to investigate the radiated spin particle's action.

Recently, we have explored some work [15] about the tunneling phenomenon for different BHs by using the above referred techniques. Here, we investigate the radiation spectrum via fermions tunneling of charged particles through the horizon of a BH having torus-like topology as well as charged dilatonic BHs and also the corresponding Hawking temperature. The graphical representation of Hawking temperature and horizon radius is also given. We discuss the effects of cosmological constant and charge on temperature as well as horizon radius. The format of the paper is the following: Section 2 provides review of the basic procedure about tunneling spectrum. In section 3, we investigate fermions tunneling spectrum to both torus-like BH and dilaton $\mathrm{BH}$ in anti-de Sitter spaces. Finally, section 4 summarizes the results.

\section{Charged Fermions Tunneling}

In order to discuss tunneling spectrum of the given BHs, we adopt a semiclassical tunneling method based on the Kerner and Mann technique [14. The Dirac equation for charged fermions in covariant coordinate system is [16]

$$
\imath \gamma^{\mu}\left(D_{\mu}-\frac{\imath q}{\hbar} A_{\mu}\right) \Psi+\frac{m}{\hbar} \Psi=0, \quad \mu=0,1,2,3
$$

where $m$ and $q$ denote mass and charge of the emitted fermion particle, respectively, $A_{\mu}$ is the 4 -potential, $\Psi$ is the wave function and

$$
D_{\mu}=\partial_{\mu}+\Omega_{\mu}, \quad \Omega_{\mu}=\frac{1}{2} \imath \Gamma_{\mu}^{\sigma \delta} \Sigma_{\sigma \delta}, \quad \Sigma_{\sigma \delta}=\frac{1}{4} \imath\left[\gamma^{\sigma}, \gamma^{\delta}\right] .
$$

The antisymmetric property of the Dirac matrices [17], i.e., $\left[\gamma^{\sigma}, \gamma^{\delta}\right]=0$ for $\sigma=\delta$ and $\left[\gamma^{\sigma}, \gamma^{\delta}\right]=-\left[\gamma^{\delta}, \gamma^{\sigma}\right]$ for $\sigma \neq \delta$, reduces the Dirac equation (2.1) in the following form

$$
\imath \gamma^{\mu}\left(\partial_{\mu}-\frac{\imath q}{\hbar} A_{\mu}\right) \Psi+\frac{m}{\hbar} \Psi=0 .
$$

The spinor wave function $\Psi$ (related to the particle's action) has two spin states: spin-up and spin-down in +ve and -ve $r$-directions, respectively. For 
the spin-up and spin-down particle solution, we assume [14]

$$
\begin{aligned}
& \Psi_{\uparrow}(t, r, \varphi, \psi)=\left[\begin{array}{c}
E(t, r, \varphi, \psi) \\
0 \\
F(t, r, \varphi, \psi) \\
0
\end{array}\right] \exp \left[\frac{\imath}{\hbar} I_{\uparrow}(t, r, \varphi, \psi)\right], \\
& \Psi_{\downarrow}(t, r, \varphi, \psi)=\left[\begin{array}{c}
0 \\
G(t, r, \varphi, \psi) \\
0 \\
H(t, r, \varphi, \psi)
\end{array}\right] \exp \left[\frac{\imath}{\hbar} I_{\downarrow}(t, r, \varphi, \psi)\right],
\end{aligned}
$$

where $I_{\uparrow / \downarrow}$ symbolize the radiated particle's action regarding spin-up/spindown, respectively. The particle's action is described by

$$
I_{\uparrow}=-\omega t+W(r)+J(\varphi, \psi)
$$

where $\omega, J$ and $W$ are energy, angular momentum and arbitrary function, respectively.

The line element for torus-like $\mathrm{BH}$ can be written in the following form

$$
\mathrm{d} s^{2}=-g(r) \mathrm{d} t^{2}+\frac{\mathrm{d} r^{2}}{g(r)}+r^{2}\left(\mathrm{~d} \varphi^{2}+\mathrm{d} \psi^{2}\right)
$$

Using the ansatz (2.5) into the Dirac equation with $E=-\imath F, E=\imath F$ and Taylor's expansion of $g(r)$ near the event horizon $r_{+}$, it follows that

$$
\begin{array}{r}
-F\left[\frac{-\left(\omega+q A_{t}\right)}{\sqrt{\left(r-r_{+}\right) g^{\prime}\left(r_{+}\right)}}+\sqrt{\left(r-r_{+}\right) g^{\prime}\left(r_{+}\right)} W^{\prime}(r)\right]+m E=0, \\
-\frac{F}{r}\left[\partial_{\varphi} J+\imath \partial_{\psi} J\right]=0, \\
E\left[\frac{-\left(\omega+q A_{t}\right)}{\sqrt{\left(r-r_{+}\right) g^{\prime}\left(r_{+}\right)}}-\sqrt{\left(r-r_{+}\right) g^{\prime}\left(r_{+}\right)} W^{\prime}(r)\right]+m F=0, \\
-\frac{E}{r}\left[\partial_{\varphi} J+\imath \partial_{\psi} J\right]=0 .
\end{array}
$$

It is mentioned here that, the condition $E=-\imath F$ holds for outgoing solutions, while $E=\imath F$ for the incoming solutions. Equations (2.8) and (2.10) imply that

$$
J(\varphi, \psi)=c_{1} e^{c_{2}(\varphi+\imath \psi)}
$$


where $c_{1}$ and $c_{2}$ are arbitrary constants. This expression can be used in Eq.(2.5) to calculate particle's action. For the massless case $(m=0)$, Eqs.(2.7) and (2.9) yield

$$
W^{\prime}(r)=W_{+}^{\prime}(r)=-W_{-}^{\prime}(r)=\frac{\omega+q A_{t}}{\left(r-r_{+}\right) g^{\prime}\left(r_{+}\right)} .
$$

Integrating this equation with respect to $r$, we get

$$
W_{+}(r)=-W_{-}(r)=\frac{\omega+q A_{t}}{g^{\prime}\left(r_{+}\right)} \int \frac{d r}{\left(r-r_{+}\right)},
$$

where $W_{+}$and $W_{-}$correspond to the outgoing and incoming solutions, respectively. This equation represents the pole at the horizon $r=r_{+}$.

\section{Charged anti-de Sitter Black Holes}

In this section, we examine fermions tunneling spectrum as radiation process from charged anti-de Sitter BHs essentially torus-like and dilaton BHs.

\subsection{Torus-like Black Hole}

In general relativity, charged rotating BHs are described by mass $M$, angular momentum $J$ and electric charge $Q$. The topology of spacelike solution of the event horizon of these BH solutions is a two-dimensional sphere $S^{2}$. It would be interesting to study such BH solutions of the Einstein-Maxwell field equations whose event horizons have topologies other than $S^{2}$, e.g., toruslike. We explore charged particles tunneling from the torus-like BH solution with negative cosmological constant.

The torus-like BH solution is characterized by the line element [18]

$$
\begin{aligned}
\mathrm{d} s^{2} & =-\left(-\frac{1}{3} \Lambda r^{2}-\frac{2 M}{\pi r}+\frac{4 Q^{2}}{\pi r^{2}}\right) \mathrm{d} t^{2}+\left(-\frac{1}{3} \Lambda r^{2}-\frac{2 M}{\pi r}+\frac{4 Q^{2}}{\pi r^{2}}\right)^{-1} d r^{2} \\
& +r^{2}\left(\mathrm{~d} \varphi^{2}+\mathrm{d} \psi^{2}\right)
\end{aligned}
$$

where $\varphi, \psi \in[0,2 \pi]$. Equations (2.6) and (3.1) lead to

$$
g(r)=-\frac{1}{3} \Lambda r^{2}-\frac{2 M}{\pi r}+\frac{4 Q^{2}}{\pi r^{2}}
$$


This line element will be singular for $g(r)=0$, i.e.,

$$
-\frac{1}{3} \Lambda r^{2}-\frac{2 M}{\pi r}+\frac{4 Q^{2}}{\pi r^{2}}=0
$$

When $\Lambda>0$, the only non-zero horizon radius is

$$
r_{h}=\frac{1}{2}\left[\Upsilon^{1 / 2}+\left\{-\Upsilon+2\left[\Upsilon^{2}+(48 / \pi \Lambda) Q^{2}\right]^{1 / 2}\right\}^{1 / 2}\right]
$$

for any $M$ and $Q$, where $\Upsilon$ is given by

$$
\begin{aligned}
\Upsilon & =\left\{\frac{(6 M)^{2}}{2 \pi^{2} \Lambda^{2}}+\left[\left(\frac{(6 M)^{2}}{2 \pi^{2} \Lambda^{2}}\right)^{2}+\left(\frac{16}{\pi \Lambda} Q^{2}\right)^{2}\right]^{1 / 2}\right\}^{1 / 3} \\
& +\left\{\frac{(6 M)^{2}}{2 \pi^{2} \Lambda^{2}}-\left[\left(\frac{(6 M)^{2}}{2 \pi^{2} \Lambda^{2}}\right)^{2}+\left(\frac{16}{\pi \Lambda} Q^{2}\right)^{2}\right]^{1 / 2}\right\}^{1 / 3} .
\end{aligned}
$$

Incorporating the fact that the line element (3.1) is not static for large $r$, it implies that the metric with positive $\Lambda$ does not describe a $\mathrm{BH}$ solution.

For $\Lambda<0$, Eq.(3.3) has two positive solutions

$$
r_{ \pm}=\frac{1}{2}\left[\Upsilon^{1 / 2} \pm\left\{-\Upsilon+2\left[\Upsilon^{2}+(48 / \pi \Lambda) Q^{2}\right]^{1 / 2}\right\}^{1 / 2}\right]
$$

as long as

$$
0 \leq Q^{2} \leq \frac{3}{8}\left(3 M^{4} / 2 \pi|\Lambda|\right)^{1 / 3}
$$

Thus, the metric has coordinate singularities at the horizon radii $r_{ \pm}$for which

$$
-\frac{1}{3} \Lambda r_{ \pm}^{2}-\frac{2 M}{\pi r_{ \pm}}+\frac{4 Q^{2}}{\pi r_{ \pm}^{2}}=0
$$

where $r_{+}$and $r_{-}$represent outer and inner horizons of the $\mathrm{BH}$. The only nonvanishing ingredient of electromagnetism in the Dirac equation is $A_{t}=$ $-\frac{4 Q}{r}$.

For semiclassical tunneling probability, the wave equation is multiplied by its complex conjugate. In this way, the part of curved route (trajectory) of particles (that originate from outside region of the $\mathrm{BH}$ and prolonged to the observer) will not supplement to the calculation of the absolute tunneling probability and can be neglected. Thus, the only sector of the wave 
(trajectory) that just provides the tunneling probability is the contour encompassing the $\mathrm{BH}$ horizon. We choose that the outgoing particle contour is in the lower half plane, so by using mathematically equivalent convention we do not multiply the contour integration by a negative sign [19].

Inserting the value of $g$ in Eq.(2.13), we obtain

$$
W_{+}(r)=-W_{-}(r)=\frac{\omega+q A_{t}}{-\frac{2}{3} \Lambda r_{+}+\frac{2 M}{\pi r_{+}^{2}}-\frac{8 Q^{2}}{\pi r_{+}^{3}}} \int \frac{d r}{r-r_{+}} .
$$

Integrating around the pole, it follows that

$$
W_{+}(r)=-W_{-}(r)=\frac{\pi \imath\left(\omega+q A_{t}\right)}{-\frac{2}{3} \Lambda r_{+}+\frac{2 M}{\pi r_{+}^{2}}-\frac{8 Q^{2}}{\pi r_{+}^{3}},}
$$

where the imaginary parts of $W_{+}$and $W_{-}$yield

$$
\operatorname{Im} W_{+}=-\operatorname{Im} W_{-}=\frac{\pi\left(\omega+q A_{t}\right)}{-\frac{2}{3} \Lambda r_{+}+\frac{2 M}{\pi r_{+}^{2}}-\frac{8 Q^{2}}{\pi r_{+}^{3}}} .
$$

Thus, the particle's tunneling probability from inside of the event horizon to outside is

$$
\Gamma=\frac{\operatorname{Prob}[\mathrm{out}]}{\operatorname{Prob}[\mathrm{in}]}=\exp \left[-4 \operatorname{Im} W_{+}\right]=\exp \left[-\frac{2 \pi\left(\omega+q A_{t}\right)}{-\frac{1}{3} \Lambda r_{+}+\frac{M}{\pi r_{+}^{2}}-\frac{4 Q^{2}}{\pi r_{+}^{3}}}\right] .
$$

We expand the action in terms of particle energy $\omega$ so that the linear order leads to the Hawking temperature. The higher order terms represent the self-interaction effects resulting from the energy conservation. Thus, the emission rate in the tunneling approach just to first order in $\omega$ retrieves the Boltzmann factor, $\exp [-\beta \omega]$, where $\beta=\frac{1}{T_{H}}[20]$. It follows that the emission rate in the high energy is proportional to the Boltzmann factor, i.e.,

$$
\Gamma \simeq \exp \left[-\frac{2 \pi \omega}{-\frac{1}{3} \Lambda r_{+}+\frac{M}{\pi r_{+}^{2}}-\frac{4 Q^{2}}{\pi r_{+}^{3}}}\right]
$$

Comparing with $\Gamma \simeq \exp [-\beta \omega]$, the Hawking temperature takes the form

$$
T_{H}=\frac{1}{2 \pi}\left(-\frac{1}{3} \Lambda r_{+}+\frac{M}{\pi r_{+}^{2}}-\frac{4 Q^{2}}{\pi r_{+}^{3}}\right) .
$$




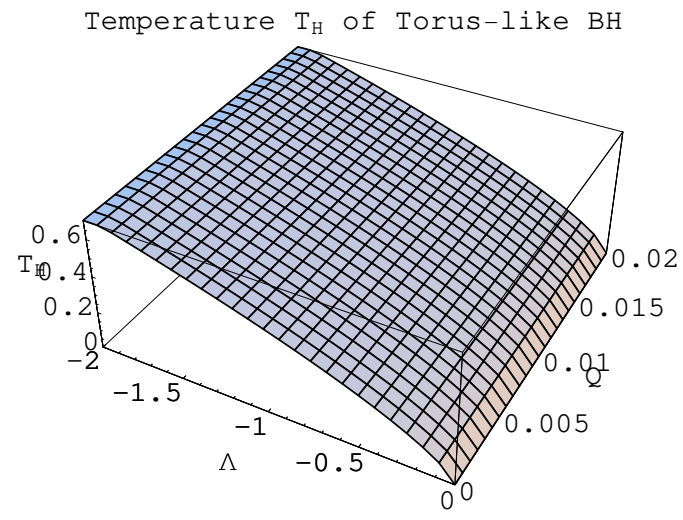

Figure 1: Hawking temperature $T_{H}$ versus cosmological constant $\Lambda$ and charge $Q$

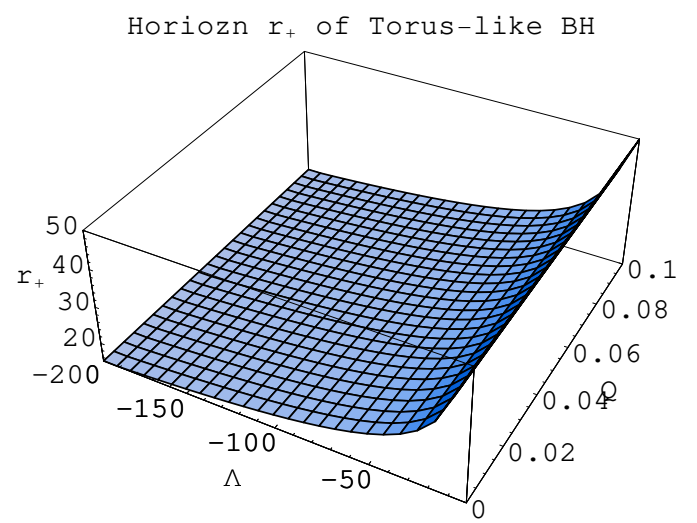

Figure 2: Horizon radius $r_{+}$versus cosmological constant $\Lambda$ and charge $Q$ 
For the massive case $(m \neq 0)$, following the same steps, we can obtain the same temperature. Thus, the behavior of massive particles near the $\mathrm{BH}$ horizon is the same as that for the massless particles.

The behavior of Hawking temperature (3.13) of torus-like BH in antide Sitter spacetime for $M=100$ (based on the cosmological constant $\Lambda$ and electric charge $Q$ ) is shown in Figure 1. This indicates that toruslike $\mathrm{BH}$ temperature increases as $\Lambda$ decreases, while charge has no effect. In this background, the location of the horizon radius can be obtained by using Eqs.(3.5) and (3.6). We also plot the horizon radius with cosmological constant $\Lambda$ and charge $Q$ for $M=100000$ displayed in Figure 2. This shows that the horizon radius decreases gradually as $\Lambda$ decreases. These results are consistent with those given in [21].

\subsection{Dilaton Black Holes}

Dilaton is characterized by a scalar field occurring in the string theory with low energy limit. Dilaton field has considerable outcome on causal structure and thermodynamics of the BH. The standard form of the line element for the static spacetime can be prescribed as 22

$$
d s^{2}=-g(r) d t^{2}+\frac{1}{g(r)} d r^{2}+f(r)^{2} d \Omega_{k, 2}^{2}
$$

where $d \Omega_{k, 2}^{2}$ is the line element for a two-dimensional hypersurface with constant curvature

$$
d \Omega_{k, 2}^{2}=\left\{\begin{array}{cc}
d \theta^{2}+\sin ^{2} \theta d \varphi^{2} & \text { for } k=1 \\
d \theta^{2}+\theta^{2} d \varphi^{2} & \text { for } k=0 \\
d \theta^{2}+\sinh ^{2} \theta d \varphi^{2} & \text { for } k=-1
\end{array}\right.
$$

The Maxwell equation, $\partial_{\mu}\left(\sqrt{-g} e^{2 a \phi} F^{\mu \nu}\right)=0$, gives the only non-zero component of the Maxwell field tensor as $F_{01}=\frac{Q e^{2 a \phi}}{f^{2}}$. The dilaton field $\phi$ can be defined as [23] $e^{2 a \phi}=\left(1-\frac{r_{-}}{r}\right)^{\frac{2 a^{2}}{\left(1+a^{2}\right)}} e^{-2 a \phi_{0}}$ with $e^{2 a \phi_{0}}=\frac{r_{+} r_{-}}{\left(1+a^{2}\right) Q^{2}}$ [21], where $\phi_{0}$ is the dilaton field at infinity and $a$ is an arbitrary parameter which represents the intensity of the coupling of the Maxwell field to the dilaton field. 
The topological BH solution can be obtained as 22

$$
\begin{aligned}
g & =\left(k-\frac{r_{+}}{r}\right)\left(1-\frac{r_{-}}{r}\right)^{\frac{1-a^{2}}{1+a^{2}}}-\frac{1}{3} \Lambda r^{2}\left(1-\frac{r_{-}}{r}\right)^{\frac{2 a^{2}}{1+a^{2}}} \\
Q^{2} & =\frac{r_{+} r_{-}}{1+a^{2}}, \quad 2 M=r_{+}+\frac{1-a^{2}}{1+a^{2}} r_{-}, \quad f=r\left(1-\frac{r_{-}}{r}\right)^{\frac{a^{2}}{1+a^{2}}} .
\end{aligned}
$$

Equation (3.16) implies that either $a=0$ or $\phi_{0}=$ constant. For $\phi_{0}=$ constant, the corresponding dilaton field is

$$
e^{2 a \phi}=\left(1-\frac{r_{-}}{r}\right)^{\frac{2 a^{2}}{1+a^{2}}} .
$$

The locations of the event horizon $r_{+}$and inner horizon $r_{-}$are

$$
r_{ \pm}=\frac{1+a^{2}}{1 \pm a^{2}}\left[M \pm \sqrt{M^{2}-\left(1-a^{2}\right) Q^{2}}\right]
$$

The Dirac equation with the spinor wave function leads to the same equations as given by Eqs.(2.7) and (2.9) with $g(r)$ defined in Eq.(3.16). The corresponding value of $J(\theta, \varphi)$ to obtain particle's action is found to be

$$
J(\theta, \varphi)=c_{3} e^{c_{4}[-f(r) \ln |\csc \theta-\cot \theta|+\imath \varphi]},
$$

where $c_{3}$ and $c_{4}$ are arbitrary constants.

The tunneling probability is given by

$$
\Gamma=\exp \left[-4 \operatorname{Im} W_{+}\right]
$$

which implies that

$$
\Gamma=\exp \left[-\frac{4 \pi\left(\omega+q A_{t}\right)}{g^{\prime}\left(r_{+}\right)}\right] \simeq \exp [-\beta \omega]
$$

Consequently, we have

$$
T_{H}=\frac{g^{\prime}\left(r_{+}\right)}{4 \pi} .
$$

The horizon radii can be obtained by using $g(r)=0$, which means that

$$
\left(k-\frac{r_{+}}{r}\right)\left(1-\frac{r_{-}}{r}\right)^{-\frac{2 a^{2}}{1+a^{2}}}=\frac{\Lambda}{3} r^{2}\left(1-\frac{r_{-}}{r}\right)^{-\frac{\left(1-a^{2}\right)}{1+a^{2}}} .
$$


Thus, we can write

$$
\begin{aligned}
g^{\prime}(r) & =\frac{\Lambda}{3\left(1+a^{2}\right)} r_{-} r^{\frac{1-a^{2}}{1+a^{2}}}\left(r-r_{-}\right)^{\frac{a^{2}-1}{a^{2}+1}}\left(1-3 a^{2}\right) \\
& +r_{+} r^{\frac{-\left(3+a^{2}\right)}{1+a^{2}}}\left(r-r_{-}\right)^{\frac{1-a^{2}}{1+a^{2}}}-\frac{2 \Lambda}{3} r^{\frac{1-a^{2}}{1+a^{2}}}\left(r-r_{-}\right)^{\frac{2 a^{2}}{1+a^{2}}} .
\end{aligned}
$$

Inserting this value of $g^{\prime}(r)$ in Eq.(3.21), the Hawking temperature can be found by using spin-up fermions tunneling from the dilaton $\mathrm{BH}$ as

$$
\begin{aligned}
T_{H} & =\frac{1}{4 \pi}\left[\frac { \Lambda } { 3 } ( M - \sqrt { M ^ { 2 } - ( 1 - a ^ { 2 } ) Q ^ { 2 } } ) \left\{1-\frac{1}{r_{+}}\left(\frac{1+a^{2}}{1-a^{2}}\right)(M\right.\right. \\
& \left.\left.-\sqrt{M^{2}-\left(1-a^{2}\right) Q^{2}}\right)\right\}^{\frac{a^{2}-1}{a^{2}+1}}+\frac{1}{r_{+}^{2}}\left(M+\sqrt{M^{2}-\left(1-a^{2}\right) Q^{2}}\right) \\
& \times\left\{1-\frac{1}{r_{+}}\left(\frac{1+a^{2}}{1-a^{2}}\right)\left(M-\sqrt{M^{2}-\left(1-a^{2}\right) Q^{2}}\right)\right\}^{\frac{1-a^{2}}{1+a^{2}}} \\
& -\frac{2 \Lambda}{3}\left(\frac{a^{2}}{1-a^{2}}\right)\left(M-\sqrt{M^{2}-\left(1-a^{2}\right) Q^{2}}\right) \\
& \times\left\{1-\frac{1}{r_{+}}\left(\frac{1+a^{2}}{1-a^{2}}\right)\left(M-\sqrt{M^{2}-\left(1-a^{2}\right) Q^{2}}\right)\right\}^{\frac{a^{2}-1}{a^{2}+1}} \\
& \left.-\frac{2 \Lambda}{3} r_{+}\left\{1-\frac{1}{r_{+}}\left(\frac{1+a^{2}}{1-a^{2}}\right)\left(M-\sqrt{M^{2}-\left(1-a^{2}\right) Q^{2}}\right)\right\}^{\frac{2 a^{2}}{1+a^{2}}}\right] .
\end{aligned}
$$

For spin-down particles, the same Hawking temperature can be recovered as given above.

The Hawking temperature (3.24) of fermions tunneling through the event horizon of dilaton anti-de Sitter BHs is found to be consistent with that given in [21]. For $\Lambda=0$, it reduces to the Hawking temperature corresponding to the charged dilatonic BHs [24]. It is worth mentioning here that the Hawking temperature for dilaton anti-de Sitter BHs with arbitrary coupling constant $a$ is independent of $k$ by using Eq.(3.22). Thus, the Hawking temperature of fermions tunneling through surfaces of event horizons of two-dimensional sphere (for $k=1$ ), two-dimensional torus (for $k=0$ ) and two-dimensional hyperboloid turns out to be the same. 


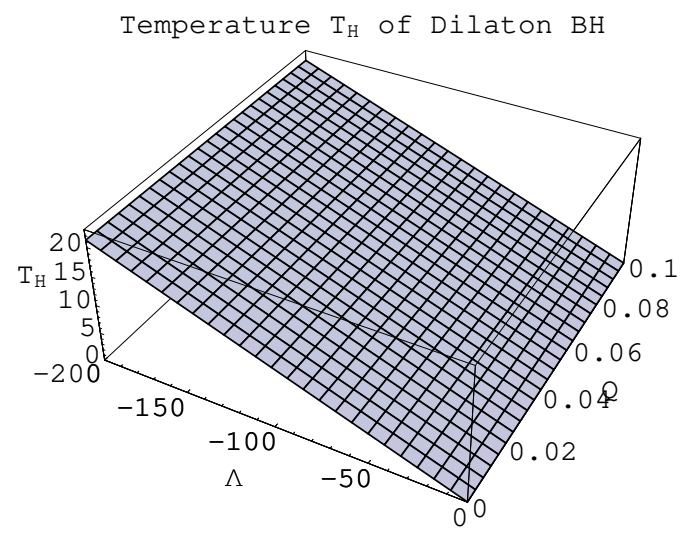

Figure 3: For $0 \leq a<1$, the Hawking temperature $T_{H}$ versus cosmological constant $\Lambda$ and charge $Q$

For $a=0=\Lambda$ and $k=1$, the line element (3.14) reduces to the RN $\mathrm{BH}$ and also the Hawking temperature $T_{H}$ (3.24) reduces to the RN BH temperature [7]

$$
T_{H}=\frac{1}{2 \pi} \frac{\sqrt{M^{2}-Q^{2}}}{\left(M+\sqrt{M^{2}-Q^{2}}\right)^{2}} .
$$

The condition for the extremal BH, i.e., $r_{+}=r_{-}$, suggests that the surface gravity and the Hawking temperature of dilaton $\mathrm{BH}$ vanish for all values of the coupling constant $a$. We have plotted the effect of $a$ on Hawking temperature. For $0 \leq a<1$ and $M=1$, the Hawking temperature (3.24) increases as $\Lambda$ decreases and hence it diverges as shown in Figure 3. For $a>1, T_{H}$ diverges which leads to the extremal $\mathrm{BH}$ case [24]. In this case, we assume $M=1$ and $a=10$ to explore $T_{H}$ which is related to the cosmological constant $\Lambda$ and electric charge $Q$. The graphical expression of $T_{H}$ is given in Figure 4. Thus, the Hawking temperature of dilaton $\mathrm{BH}$ increases as $\Lambda$ decreases but charge increases. This leads to the fact that in anti-de Sitter spacetime, the negative value of $\Lambda$ represents the force of attraction supporting gravitational collapse. Thus, the temperature increases and finally diverges.

The horizon equation $g(r)=0(g(r)$ is given in (3.16) $)$ is found to be

$$
\begin{aligned}
& -\frac{\Lambda}{3} r^{4}+k r^{2}+r\left[-\left(M-\sqrt{M^{2}-\left(1-a^{2}\right) Q^{2}}\right)\right. \\
& \left.-k \frac{1+a^{2}}{1-a^{2}}\left(M-\sqrt{M^{2}-\left(1-a^{2}\right) Q^{2}}\right)\right]+\left(1+a^{2}\right) Q^{2}=0
\end{aligned}
$$




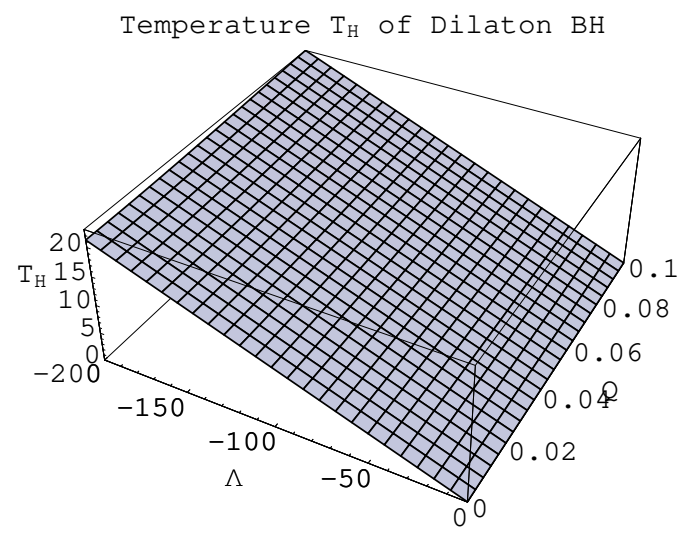

Figure 4: For $a>1$, the Hawking temperature $T_{H}$ versus cosmological constant $\Lambda$ and charge $Q$

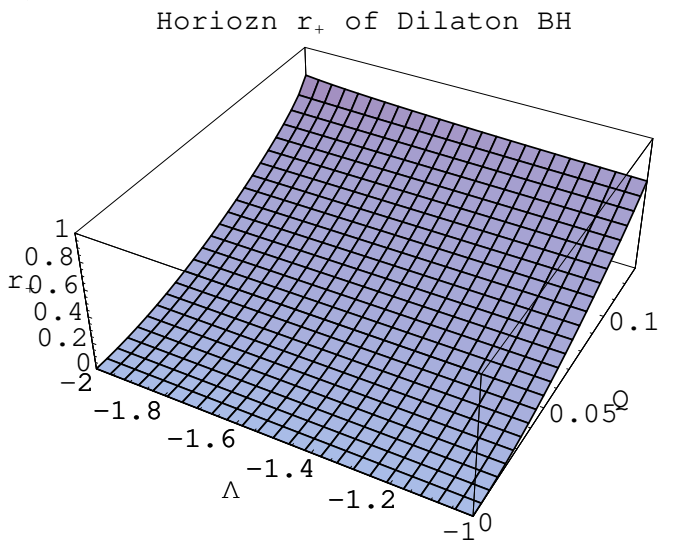

Figure 5: For $a>1$, the horizon radius $r_{+}$versus cosmological constant $\Lambda$ and charge $Q$ 
by assuming that the dilaton field takes its extreme value, $\left(1-\frac{r_{-}}{r}\right)^{\frac{2 a}{\left(1+a^{2}\right)}}=$ $\frac{r_{+} r_{-}}{\left(1+a^{2}\right) Q^{2}}$. When the coupling parameter $a=0($ or $\phi=$ constant $=0)$, the potential of the dilaton gravity theory is reduced to the cosmological constant. Also, we formulate the graphical behavior of the horizon radius (3.26) with cosmological constant $\Lambda$ and charge $Q$ in the dilaton field extremal limit, $\phi=\phi_{0}$, shown in Figure 5. We have taken $M=1, a=10$ and for all $k=-1,0,1$, it turns out that the horizon radius increases in a gradual manner as $\Lambda$ decreases. Thus for $a>1, \Lambda$ decreases while the horizon radius shows opposite behavior with that given in [21] and leads to the RN-de Sitter case [25]. The $\mathrm{BH}$ still radiates but does not vanish, i.e., as horizon radius increases, the charge also increases.

This shows rapid expansion in the horizon radius of the dilaton BHs which proves the fact that when $\phi=\phi_{0}=0$, the charged dilaton BHs lead to the RN-de Sitter BH solution [25]. Thus, the dilaton field at infinity does not support the phenomenon of BH evaporation [24]. For $a=1$, Eqs.(3.24) and (3.26) imply that there exists no graphical representation of the Hawking temperature as well as horizon radius (extremal case). For $0 \leq a<1$, the graphical illustration of horizon radius provides that $\Lambda$ and $Q$ constantly affect the horizon radius and it vanishes for all $\Lambda \leq-0.1$ and $0 \leq Q \leq 0.2$.

\section{Discussions}

Semiclassically, from a pair of particles (created in the vicinity of the event horizon due to vacuum fluctuations), a positive energy particle has the ability to tunnel outside the horizon (contradicts classical approach), while a negative energy particle tunnels inward. Thus quantum mechanically, horizon plays a role of two way energy barrier for a pair of positive and negative energy particles. We have considered tunneling probabilities for both incoming as well as outgoing particles. Relating these tunneling probabilities with the expression, $\exp [-\beta \omega]$, we can recover the corresponding Hawking temperature for these BHs at event horizons.

To this end, we have used Kerner and Mann semiclassical fermions tunneling process through WKB approximation. We have explored the process of charged fermions tunneling from charged torus-like and dilaton anti-de Sitter BHs. We have recovered the corresponding Hawking temperatures of these BHs through this tunneling process. The graphical behavior of horizon radius and temperature is also given. For the dilaton $\mathrm{BH}$ solution, the hori- 
zon radius and Hawking temperature show the non-trivial dependence on the dilaton coupling parameter $a$. This implies that the Hawking temperature increases exponentially with the coupling parameter $a$.

\section{Acknowledgment}

We would like to thank the Higher Education Commission, Islamabad, Pakistan, for its financial support through the Indigenous Ph.D. 5000 Fellowship Program Batch-IV.

\section{References}

[1] Hawking, S.W.: Phys. Rev. Lett. 26(1971)1344.

[2] Bekenstein, J.D.: Phys. Rev. D 7(1973)2333; ibid. 9(1974)3292.

[3] Hawking, S.W.: Comm. Math. Phys. 43(1975)199.

[4] Damour, T. and Ruffini, R.: Phys. Rev. D 14(1976)332.

[5] Kraus, P. and Wilczek, F.: Nucl. Phys. B 433(1995)403; 437(1995)231.

[6] Keski-Vakkuri, E. and Kraus, P.: Phys. Rev. D 54(1996)7407.

[7] Parikh, M.K. and Wilczek, F.: Phys. Rev. Lett. 85(2000)5042.

[8] Parikh, M.K.: Phys. Lett. B 546(2002)189.

[9] Parikh, M.K.: Gen. Relativ. Gravit. 36(2004)2419 [Int. J. Mod. Phys. D 13(2004)2351].

[10] Hemming, S. and Keski-Vakkuri, E.: Phys. Rev. D 64(2001)44006.

[11] Medved, A.J.M.: Phys. Rev. D 66(2002)124009.

[12] Zhang, J. and Zhao, Z.: Phys. Lett. B 618(2005)14.

[13] Zhang, J. and Zhao, Z.: Mod. Phys. Lett. A 20(2005)1673.

[14] Kerner, R. and Mann, R.B.: Class. Quantum Grav. 25(2008)095014. 
[15] Sharif, M. and Javed, W.: J. Korean Phys. Soc. 57(2010)217; Astrophys. Space Sci. 337(2012)335; J. Exp. Theor. Phys. 114(2012)933; Charged Fermions Tunneling from Regular Black Holes, J. Exp. Theor. Phys. (to appear, 2012); Eur. Phys. J. C 72(2012)1997.

[16] Zeng, X.X. and Yang, S.Z.: Gen. Relativ. Gravit. 40(2008)2107.

[17] Gillani, U.A. and Saifullah, K.: Phys. Lett. B 699(2011)15; Gillani, U.A., Rehman, M. and Saifullah, K.: JCAP 06(2011)016; Rehman, M. and Saifullah, K.: JCAP 03(2011)001.

[18] Huang, C.G. and Liang, C.B.: Phys. Lett. A 201(1995)27.

[19] Kerner, R. and Mann, R.B.: Phys. Lett. B 665(2008)277.

[20] Kerner, R. and Mann, R.B.: Phys. Rev. D 73(2006)104010.

[21] Ghosh, T. and SenGupta, S.: Phys. Lett. B 696(2011)167.

[22] Gao, C.J. and Zhang, S.N.: Phys. Lett. B 612(2005)127.

[23] Liu, C.Z., Zhang, J.Y. and Zhao, Z.: Phys. Lett. B 639(2006)670.

[24] Chen, D.Y., Jiang, Q.Q. and Zu, X.T.: Class. Quantum Grav. 25(2008)205022.

[25] Gao, C.J. and Zhang, S.N.: Phys. Rev. D 70(2004)124019. 